\title{
Meter Placement in Power System Network-A Comprehensive Review, Analysis and Methodology
}

\author{
Ramesh Lekshmana ${ }^{1}{ }^{(}$, Sanjeevikumar Padmanaban ${ }^{2, *} \mathbb{( D}$, Sagar Bhaskar Mahajan ${ }^{3, * \mathbb{D}}$, \\ Vigna K. Ramachandaramurthy ${ }^{4}\left(\mathbb{D}\right.$ and Jens Bo Holm-Nielsen ${ }^{2}$ \\ 1 GREEN 9 (Energy Efficiency Research Group), Department of Electrical and Electronics Engineering, \\ Dr. M.G.R. Educational and Research Institute University, Chennai 600095, India; proflramesh@green9.org \\ 2 Center for Bio-energy and Green Engineering, Department of Energy Technology, Aalborg University, \\ 6700 Esbjerg, Denmark; jhn@et.aau.dk \\ 3 Department of Electrical Engineering, Qatar University, 2713 Doha, Qatar \\ 4 Institute of Power Engineering, Department of Electrical Power Engineering, Universiti Tenaga Nasional, \\ Kajang 43000, Malaysia; vigna@uniten.edu.my \\ * Correspondence: san@et.aau.dk (S.P.); sagar25.mahajan@gmail.com (S.B.M.)
}

Received: 19 October 2018; Accepted: 13 November 2018; Published: 16 November 2018

\begin{abstract}
The blackout in North India due to the failure of the central grid has led to the need for intelligent power system state estimation, where optimal location is necessary to understand. State estimation meter placement plays a major role in the smart operation of a modern distributed power system. A literature review of the different algorithms incorporated for the determination of minimal number of meters required for the measurement of real time measurements is presented for the power system and distribution system state estimation, including smart meter location.
\end{abstract}

Keywords: distribution system; optimal meter placement; state estimation of power system; smart meter

\section{Introduction}

Effective control and operation is dependent on the accuracy of the information observed from the state estimator regarding the considered power system. In reference [1], assessment of electric power systems using Monte Carlo Methods for reliable assessment is presented. Reliable data is provided from the tele-metered measurement data obtained due to the state estimator operation to the power system control center. The data collection of real time measurement is with the help of meters (RTU), placed at various buses. The minimal planning of meters for optimal measurement is called meter placement (MP) problem. It is observed that some electricity blackouts through the world were obtained, due to the measurement error and non-availability of state estimator. The complexity in the modern power system arises due to the addition of distributed generation near the customer end and the bidirectional power flow created challenges in control and protection. In the present power distributed distribution system management system, it is mandatory to have a smart intelligent state estimator with optimal measurement system to provide uninterrupted power supply to the end users. The objective function for the meter placement problem initiated in the year 1975 and several algorithms were developed over a period of 25 years by eminent professors around the globe. The current system meter placement problem classifies as Distribution System MP, Optimal Smart MP, Power Quality MP, Harmonic MP, Voltage Sag MP, PMU MP and Switch MP. This study carries out a review on power system state estimation MP, Distribution System state estimation MP and Optimal Smart MP. The readers can develop knowledge on various algorithms developed by the eminent researchers on MP. The session continues with the development of mathematical and intelligent algorithms on power system state estimation MP. 


\section{Power System SE Meter Placement}

The Park et al. [2] developed Addition and Elimination Algorithm in 1975, to design a reliable measurement system for SE to reduce the investment cost, maintaining SE accuracy. To make the most efficient use of the approach required minimizing the formulated performance index (PI), to limited system constraints, which consider the probability of measurement failures. Here, the least square error estimator was used for the optimization. On testing for IEEE 14 bus system, observability with high accuracy for any single outage in the network was obtained with marginal reduction in the investment cost and critical redundancy rate. The limitation is its incompatibility in the presence of large number of assumed measurements, due to the excessive computation time. Celik et al. [3] developed the Covariance Matrix Algorithm in 1994, which had the variance of the SE errors utilized as indices to improve the accuracy and robustness of the SE solutions. The covariance matrix (C) composed of the variance of the SE errors, is used to formulate the objective function that helps in the identification of the location of additional measurements. Results obtained from the tests on systems considered like IEEE 14, 30, 57 and 118 bus systems had the SE solutions improved with the addition of the measurements. It helps in obtaining unique numerical solution of the observable islands along with a summarized list of candidate measurements for those state variables that had a poor accuracy calculated. The limitation is the high capital cost on the metering device along with the accuracy of the State Variables calculated for the new boundary buses compromised because of insufficient measurements.

Abbasy, [4] developed the Neural Network Algorithm in 1996 for an on-line Power System State Estimation (PSSE), utilizing the characteristic learning ability of the neural network for the determination of the measurement meeting a pre-specified SE PI (State Estimation Performance Index), evaluated through the covariance matrix formulated from the errors of the estimated state vector along the computation period which are the primary constraints the monitoring and control by on-line. Tested on a 6-bus power system generating a set of 90 input-output patterns of which a subset of $30 \mathrm{I} / \mathrm{O}$ patterns were used for the trainng of ANN and another subset of $5 \mathrm{I} / \mathrm{O}$ patterns were used for testing after the training, resulting in a satisfactory performance leading to two hidden layers along with 10 neurons in each hidden layer with a pre-specified accuracy level of 0.005 at a running time of $1 \mathrm{pu}$. The only limitation is the inability to detect bad data and system topology. Abur et al. [5] developed the Linear Programming (LP) Algorithm in 1999, aiding in maintenance of observability even in the absence of network branches, in just two steps. The first step was LP based meter placement being used in the determination of the number of additional measurements required to push the system into critical state leading to reduced observability, but this is liable to branch outages. The second computes the optimal number along with the type of measurements required for the maintenance of the observability of the system against single branch outages. Tests of the proposed algorithm on IEEE 30 and 70 bus systems have been successful.

Mori et al. [6] developed Tabu Search (TS) Algorithm in 1999 based on the hill climbing method, a local search, enhancing the observability of the topology along with the relationship between network configuration and meter placement. Tests on IEEE 57 and 118 node systems, considering redundancy value set to 1.31 and 1.45 respectively, resulted in $1.426 \times 106$ and $4.238 \times 1014$ combinations respectively with CPU time 4.34 times faster than Simulated Annealing and Genetic Algorithm. The tested result for IEEE 118 node with SA, GA and TS is represented in Table 1.

Table 1. Comparative analysis of algorithms.

\begin{tabular}{cccc}
\hline Methods & CF $^{*}$ & SD $^{*}$ & CPU Time \\
\hline SA & $4.876 \times 10^{36}$ & $5.39 \times 10^{35}$ & 9.12 \\
GA & $4.802 \times 10^{36}$ & $2.07 \times 10^{35}$ & 1.99 \\
TS & $4.888 \times 10^{36}$ & 0 & 1.00 \\
\hline \multicolumn{4}{c}{${ }^{*}$ Cost Factor $(\mathrm{CF})$ and Standard Deviation (SD). }
\end{tabular}


The traditional TS Algorithm is run a number of times, while for the initialization of each search in the algorithm, the best solution obtained from all previous executions is used. Nikolaos et al. [7] developed the Recursive Tabu Search Algorithm in 2012, to reach a solution for the OPP problem by verifying observability of the network with a numerical algorithm. Tests on IEEE 14, 30, 57 and 118-bus systems resulted in the determination of optimal number of meter placements unlike the other methods, which either provided the same minimum number of meter placements or sometimes a higher number. Abur et al. [8] developed the Integer Programming (IP) and Sparse Triangular Factorization (STF) Algorithm in 2000, to numerically yield measurement configuration withstanding branch outage of any single branch or even in case of loss of single measurement without losing network observability, based on STF and the measurement Jacobian aiding in easy incorporation in the existing state estimators. The algorithm is a two-step procedure, the first assisting in selection of candidates by STF and modified Jacobian and the second aiming at optimal selection. From the tests on IEEE 30 bus system, with injection measurements at buses 6,9 and 11 and flow measurement in branches 27-29, satisfactory network observability is observed during any single line outage or even in the absence of any single measurement, with the inclusion of the above mentioned additional measurements. Bilgehan et al. [9] further extended the research in 2011, developing an alternative approach applicable for large scale power system aiming at numerical observability, achieving higher efficiency with the placement of multiple measurement values in each iteration, restoring in a lesser number of iterations in comparison to earlier methods. The Simulated Annealing (SA) Algorithm was developed to reduce the installation cost and distribution of the measuring device with the objective function minimized using SA. The constraints on the objective function are the cost function, observability requisite and reliability requisite. The algorithm proposed in the paper, on testing on IEEE 30 bus system resulted in greater reliability with reduced computational time, obtaining a significant number of configurations with neither critical measurements nor sets.

Souza et al. [10] developed the Genetic Algorithm (GA) in 2005, to be incorporated for any topology that the network may experiment. The advantage is that the trade-off between investment cost and reliability of the SE process under any topology scenario can be achieved. It involves the formulation of a fitness function aimed at reduction of the cost of metering system, in the unavailability of critical measurements. The performance requirements are the constraints on the objective function. Tests on the ELETROPAULO network with 61 buses with 74 branches, with GA chromosome depicted by a vector of 209 genes, where 148 genes involved with possible power flow measurement locations and 61 genes involved with possible power injection measurement locations, resulted in desired solutions with reduced convergence time. Ashwani et al. [11] justified in 2005, considering IEEE $14,18,30$ and 145 bus systems, the increased estimation accuracy and reduced computational time in comparison with the Sequential Method and Complete Enumeration (CE) respectively, used for static estimation of the considered power system in the presence of harmonic sources through the determination of optimal meter placement.

Jian et al. [12] in 2006, successfully used the TOMLAB Optimization Tool Box for solving the objective function formulated using Integer Programming for IEEE 57 and 118 bus systems Nabiletal et al. [13] developed Augmented Bus Merging (ABM) Method in 2009, to determine the optimal measurement unit placement in the presence of one or multiple measurement unit loss, keeping the entire system observable. On testing on IEEE 14, 30, 57 and 118 bus systems, the proposed method has been proved to be much better than the Individual Bus Merging (IBM) Method and Proposed Local Redundancy (LR) Method. The advantage of the method is the effect of the meter cost on their optimal number and locations Sodhi et al. [14] developed a Multi-Criteria Decision Making Approach in 2010, to place the measurement units in a number of stages for a considered period of time to ensure complete power system observability even when subjected to a branch outage or a measurement unit failure. The advantage is the installment of the measurement devices in stages, thus distributing the capital cost over a given time period, owing to the relatively high cost of the meters. Mohammad et al. [15] developed in 2012 an algorithm for the simultaneous optimization of 
both, meter placement along with the communication infrastructure required for the state estimation program, realizing the objective with the formulation of two power system optimization problems initially which are then simultaneously formulated in a single problem through a co-optimization method based on GA. The first is the formulation of SE meter placement and the second is the Communication Infrastructure Designing problem. The unique feature of this algorithm is the use of double genes in this algorithm.

Yuttana et al. [16] developed Key Cutting Algorithm in 2013, for solving the problem of measurement placement for the power system state estimation, by representing measurement installation in power system through " 0 " and " 1 ". The concept of similarity probability of a key to a key set is used for the new measurement installation. One of its features is the inclusion of observability and critical measurements in the objective function. The tests have resulted in obtaining best results with reference to the percentage of the best hit on using the algorithm for conditions with a low probability factor. Xiao Li et al. [17] developed Gossip based Gauss Newton Technique in 2013 for the evaluation of a power system through a fully decentralized adaptive re-weighted state estimation scheme by means of network gossiping, mitigating the effect of bad data through online updateable error variances and re-weighting their contributions adaptively for state estimation, aiding in global state estimation being tracked robustly through the use of near-neighbor communications in each area. The advantage of this technique over others is the incorporation of the communication model which adapts to changes due to reconfiguration and resilient to random failures provided the communication network is connected. Yang Weng et al. [18] developed graphical model presentation for State Estimation Technique in 2013, to account for uncertainties due to advanced technology such as penetration of green energy distribution in intelligence and plug-in electric vehicles. It discovers and analyzes unstructured information. Simulation results have notably improved performance of state estimation over the conventional deterministic approach when computational time and accuracy are considered and have enabled scalability of state estimation for the non-linear computational time.

Sourav et al. [19] developed an evolutionary search technique derived from Improved Particle Swarm Optimization and Gravitational Search Algorithm in 2013, to evaluate the Static State Estimation Problem in terms of optimization. On testing on five IEEE standard systems, the results obtained are better than those obtained by Weighted Least Square State Estimation and State Estimation obtained from Particle Swarm Optimization. Later, research has been carried out by Tungadio et al. [20] to incorporate Genetic Algorithm in Particle Swarm Optimization. Bhuvana et al. [21] proposed the best algorithm in 2014 for determining the observability of the considered power system with the help of PMU placement. The minimum numbers of phasor measurement units are obtained by using Improved Fruit Fly Optimization Method and it is tested on IEEE 14, 24, 30, 57 and 108 bus systems. The tested results are validated with the existing study.

A comparison of the number of studies per year on meter placement on Power System State Estimation and an evolution for algorithms has represented in Figure 1. Meter placement for PSSE was first studied in the year 1975. Nine different approaches [22] proposed until the year 2006 was discussed in detail by the authors, considering system observability. The extension of the work until the year 2015 with more than fifteen algorithms is summarized in this work.

A large number of works done between (1999-2001) with power system RTU placement and (2009-2014) with optimal PMU placement, the work still continues with encompassing new approaches such as distributed generation, smart grid, micro grid etc. The session continues with a review on distribution system measurement placement. 


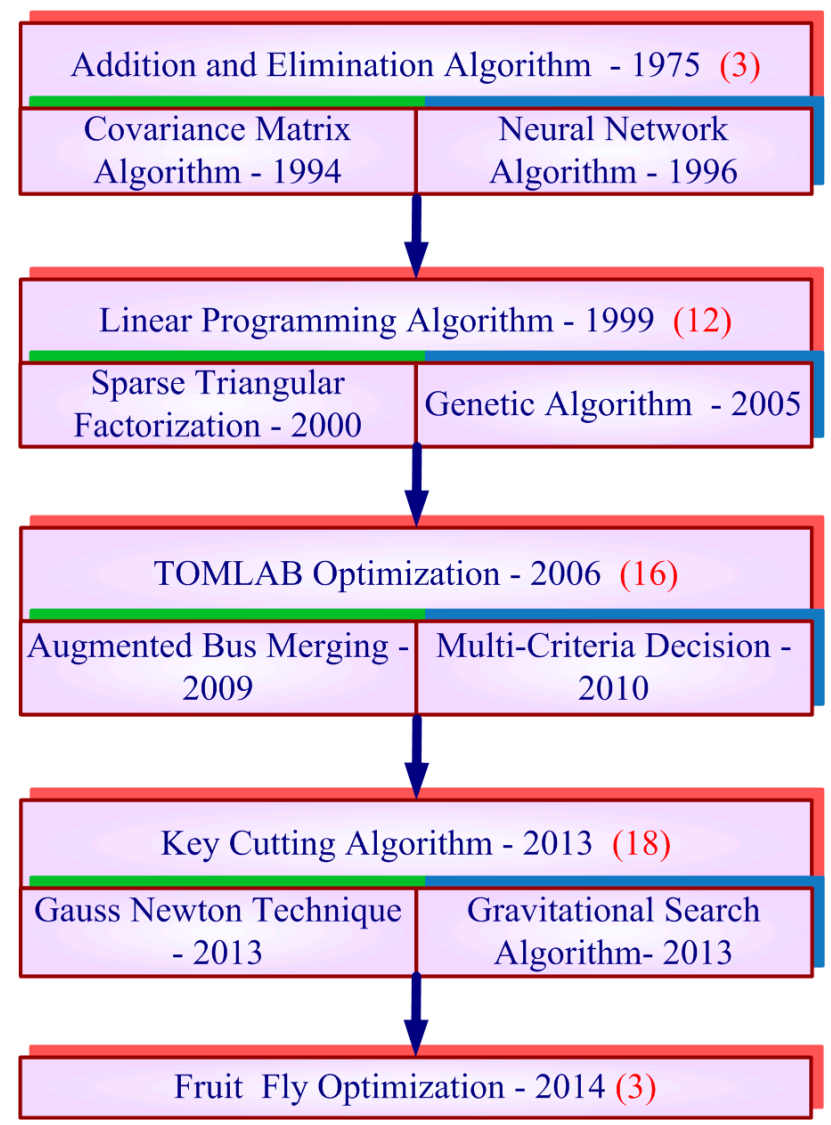

Figure 1. Evolution of online Power System State Estimation (PSSE) Meter Placement Algorithms.

\section{Meter Placement of Distribution System State Estimation}

Baranetal [23] proposed the rule-based meter placement algorithm in 1996, to determine estimation of the data needed with improved accuracy for the feeder automation functions by identifying data required for the real-time monitoring and control of the considered distribution systems. Souza et al. [24] proposed Genetic Algorithm based meter placement technique in 2003, enabling features such as varied network configurations, utilization of pseudo measurements, already installed meters and remote terminal units among others, accounting to its flexibility. It is used to obtain a trade-off between real-time monitoring capability and investment costs with the aim of planning adequate metering systems as the problem statement. Wang et al. [25] proposed a Reverse Branch Current Meter Placement Impact in 2004, based on meter placement impact on the system through three phase branch current state estimation algorithm. The magnitude and the phase angle of the branch current are represented by the state variables. The results provided are satisfactory, with the scope of research being extended to more areas particularly three, namely more topologies, more detailed models and test on an actual system having both AMR and other real measurements.

Das et al. [26] proposed Meter Placement based on ANN Selection in 2005, to obtain a combination with reduction in the number of sensors and measurement units placed based on a simple rule based algorithm, maintaining at least the previous accuracy of the bus voltage estimation. The uniqueness of the scheme is the use of multiple ANN, thus leading to significant improvement in the accuracy of estimation. It is to be considered that accuracy of the estimation depends on the number of ANN's used as well as the location of the meters. Shafiu et al. [27] proposed the Multiple Load Flow Voltage Measurement Technique in 2005, for the identification of the potential points at location of voltage measurements for the state estimation, thus minimizing the voltage standard deviation of the unmeasured bus bars. It improves the observability of the network, by addressing the problems of traditional transmission meter placement methods. Muscas et al. [28] proposed Dynamic Programming 
with Monte Carlo [1] Statistical Approach in 2006, to select the optimal number and position of the measurement devices required for operation, management and control issues, like energy dispatching and protection coordination, thus improving the observability of the system, limited to predetermined levels of accuracy and reliability. A heuristic optimization technique is used for the determination of the optimal solution for meter placement and the Monte Carlo Approach, to consider the random variations in the load and to eliminate the drawback in this approach, developed a modified technique [29] by calculating the propagation value of the uncertainty variable from measured to evaluated data initiating from suitable uncertainty models of each system element.

Cecchi et al. [30] developed a Reconfigurable Distribution of Automation and Control Technique in 2007 for a laboratory, capable of keeping a check on a maximum of 16 user-selected locations, with the recording and display of real time measurements like current, voltage, power waveforms and operating devices such as digital relays, to reconfigure the network structure which enables the SE. Bignucolo et al. [31] developed Variance Moment Genetic Algorithm Meter Placement Technique in 2007 to determine a faster calculation of the active network performances along with detecting possible problems in the estimated voltage uncertainty, defining a suitable parameter and variance moment. The uniqueness of the technique is the consideration of network structure along with the features of consumptions and generations, according to the time-variable probabilistic value of loads demand and generators production respectively. Ramesh et al. [32] developed a Particle Swarm Optimization (PSO) algorithm for the initial setting up of the metering scheme in 2008, to determine the quantity, position and type of meters to be placed in a system to obtain its observability. For the same meter placement problem, Moradi and Kennady developed the binary version of PSO [33,34] to place the location of meter. Accounting to the randomness of the proposed PSO Algorithm, a modified algorithm is incorporated, restricting the range of the particles to [0,1] with respect to their position though, the calculated velocity is in the normal procedure.

Singh et al. [35] proposed the Bivariate Chebyshev bound Measurement Placement technique in 2009, to improve the standard of the estimation of voltage and angle across the given network. The proposed scheme is dependent on the sequential improvement of the bivariate probability index governing relative errors in voltage and angle at each bus. It is difficult to get the accurate estimated value, if the state estimator is designed inefficiently. Ramesh et al. [36] in 2012 developed an intelligent MP and hybrid SE estimator to obtain optimal operation of a power distribution system. The algorithm is developed with the help of Differential Evolution and particle swarm optimization. The proposed algorithm is verified with the IEEE and TNEB benchmark systems. The real time measured value has been monitored via the proposed identified location nodes with a random noise level of $\pm 20 \%$ at selected nodes. The representation of cost function of Particle Swarm Optimization (PSO) and Differential Evolution (DE) is shown in Figure 2.

The obtained outputs of the proposed algorithms are analyzed to verify the credibility and superiority of the proposed algorithm. The results concluded that a swarm tuned ANN estimator through DE meter placement is best suited for the estimation of voltage with different noise levels with improved accuracy. The author suggested the inclusion of the impact of DG penetration in a distribution system for meter placement and state estimation as future work.

Daniel et al. [37] proposed a liberalized three-phase, distribution type of state estimation algorithm in 2013, for application in Smart Distribution system, having the flexibility to be used for even un-balanced three-phase as well as single phase cases. The designed estimator has a complex formulation and is thought to include synchronized phasor measurements into distribution SE. Yu Xiang et al. [38] 2014 proposed the probabilistic approach for an optimized MP along with a state-estimator. This is operated to offer a guide for grid operators to manage medium voltage in distribution grids. The observability of state estimator is validated through Monte Carlo optimization of the measurement with minimum measured points. The case study results are presented for the European distribution grid with the following factors, namely including installation cost of measurement systems, absence of measurement data and small voltage deviation across medium 
voltage grids. Damavandietal., in 2015 [39] concentrated on next generation distribution systems with an optimal solution for placement of voltage magnitude meters and phasor measurement units, very few in number, for state estimation. The greedy approach and sub-modular saturation algorithm are used to provide a near-optimal solution for placing meters. From the numerical analysis on three different active distribution systems it can be observed that the sub-modular saturation algorithm is much better than the greedy algorithm.

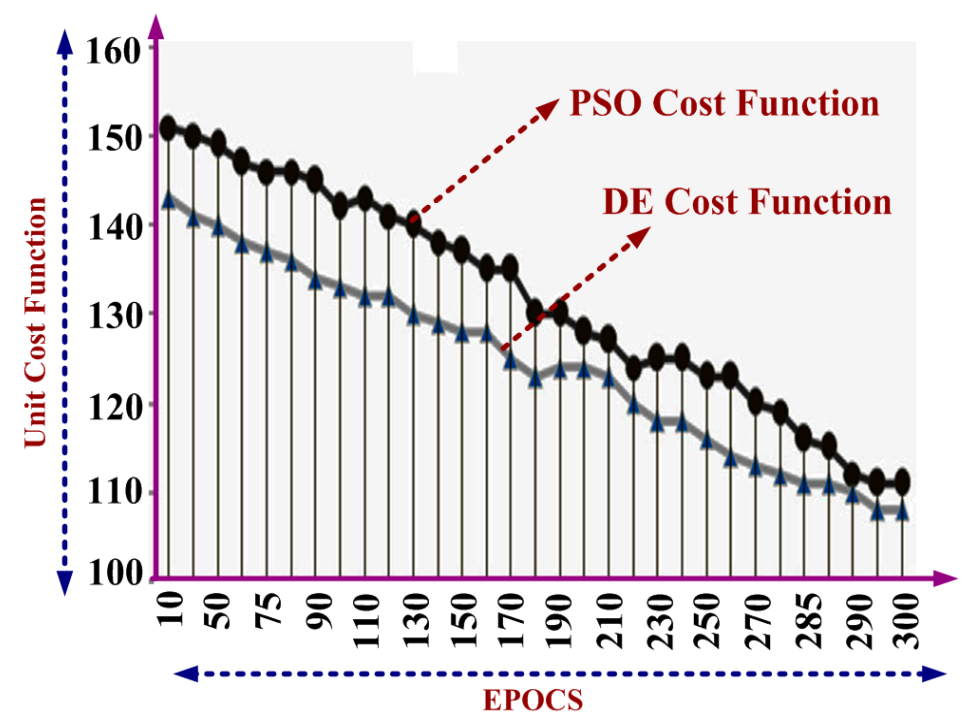

Figure 2. Cost Function Comparison for Particle Swarm Optimization (PSO) and Differential Evolution (DE).

Work on distribution system meter placement started in the year 1994. A limited amount of research work was carried over from 1994 to 2006. There is a gradual increase in the research on distribution meter placement from the year 2007, after the DG concept with bidirectional power flow. The detailed discussion on meter placement algorithms in distribution system is discussed with the author [40] until the 2008. As stated in the review of meter placement, there are seven key issues associated with Distribution System Meter Placement State Estimation. The key issues are Optimality, Versatility, Computational Efficiency, Reliability, Ability to Compute, State Estimation and Bad Data Detection.

The evolutions of meter placement algorithms are as follows: Rule based meter placement, Genetic Algorithm, Reverse Branch Current Meter Placement, Multiple Load Flow (MLF) Artificial Neural Network, Dynamic Programming, Monte Carlo Statistical Approach, Variance Moment GA, Particle Swarm Optimization, Bivariate Chebyshev bound Measurement Placement, Differential Evolution, Greedy Approach and Sub-modular Saturation Algorithm. The review on optimal smart meter placement algorithms which is necessary for operation and control of distributed distribution system is presented in the next session.

\section{Optimal Smart Meter Placement}

Liu et al. [41] proposed an algorithm in 2012, to design a basic inner structure for the measurement devices to integrate heterogeneous measurements and ensure the degree of excellence of the state estimation in varied operating conditions with minimum cost as the constraint. The measurements received from the substation and pseudo-measurements were integrated with synchro-phasor and smart meter measurements. Genetic Algorithm is used for optimization purposes, where a suitable optimization function is chosen for every optimization task. The proposed approach in this paper is specifically suited for active and smart grids. Pegoraro et al. [42] proposed an algorithm in 2012, to account for the variability with uncertainty in the behavior of load and renewable generation, a concept based on renewable energy, at different instants for a real time network, a critical aspect for 
the improved accuracy of the state estimation. The performance of the state estimation for a given topology are a measure of two synthetic indices, dependent on the theoretical covariance matrix of the overall estimation equation obtained after including smart meter measurement and phasor measurement unit measurements into the estimation through post processing.

Liu et al. [43] proposed an algorithm in 2013, to develop a measurement system which can withstand exposure to faults or even in the event of malfunction of the connected measurement devices on inclusion of $\mathrm{N}-1$ robustness and $\mathrm{N}-1$ degradation robustness as additional constraints for the optimization function. Liu et al. [44] extended an algorithm in 2014, to optimally place the measuring instruments only with the knowledge of non-Gaussian probability density distribution of even the non-monitored distributed generation, taking into consideration the uncertainty and also the $\mathrm{N}-1$ robustness and N-1 degradation robustness. The standard Weighted Least Square Estimation is considered for the formulation of State Estimation and Genetic Algorithm for optimization. To include the lack of detailed information, non-Gaussian distribution of the distributed power generation has also been taken into consideration, through inclusion of Gaussian mixture model by means of Gaussian component combination method. Here, the DG power injection plays the role of temporal pseudo measurements. The optimization is dependent on the Monte Carlo method and Genetic algorithm, which is then performed to get the robust metering infrastructure for $\mathrm{N}$ and $\mathrm{N}-1$ robustness. The probability density distribution is not based on Gaussian distribution, thus leading to the use of GMM, hence, introducing GCCM to incorporate non-Gaussian pdf data into state estimation and meter placement optimization. The optimization is validated and compared through Monte Carlo simulations. The optimization function is based on the measurement configuration cost with the accuracy index being one of the major criterions. The procedure has been tested on a UK 16 bus test network with the different types of load and distributed generation varying from domestic loads, commercial loads, industrial loads, wind turbine and photovoltaic setups and the results are shown in Table 2.

Table 2. Location of Smart Meter and PMU.

\begin{tabular}{cccc}
\hline Robustness & SM Location * & PMU Location * & Relative Cost \\
\hline $\mathrm{N}$ & 10 & 2,10 & 2.2 \\
$\mathrm{~N}-1$ & $5,7,8,11,16$ & $2,10,12$ & 4 \\
$\mathrm{~N}-1$ & $7,11,12$ & $2,4,10$ & 3.6 \\
Degradation & & & \\
\hline \multicolumn{4}{c}{ * Smart Meter (SM), Phasor Measurement Unit (PMU). }
\end{tabular}

It is shown that for the optimal meter placement, $\mathrm{N}-1$ robustness leads to increased number of installation of meters, thus leading to higher costs. However, for the maximum value performance indices, the maximum deviation indices are significantly much lower in the case of N-1 robustness. The observation results based on GCCM are not in sync with those obtained with the Monte Carlo method. However, the fact that they are significantly close to the Monte Carlo reference results in justification of the possibility of using the GMM approach.

Research has been carried for obtaining improved accuracy of the estimation in low voltage grids where, in case of improved state estimation, the relative estimation error distribution for state variables is used as the initial measure of the estimator's accuracy [45]. Furthermore, the estimation error can be reduced economically by incorporating more real-time measuring devices at the secondary substations by choosing appropriate network nodes to be measured to ensure the observability of the entire system [46]. Research has been further extended to the wireless communication technology enabling the effective data transfer between smart systems. Jamming attacks disrupting the reliability of the wireless communication can be effectively monitored and controlled with the exploitation of local controller switching [47]. Inclusion of a platform called the middleware management, acting as the logic layer, used to implement algorithms and protocols making the wireless mesh architecture flexible [48]. The present research has to look seriously at the development of smart meters and finding the optimal 
location with minimum cost, which is essential for the active operation of smart cities. The session continues with discussion and the comparative result of algorithms. In reference [49], to locate all fault type in a distribution system, a new methodology called the impedance-based technique is presented. Series impedance of the distribution lines is utilized to form an impedance matrix. Using the least square methodology along with impedance matrix and voltage phasors (pre-fault and during-fault) at buses are used to estimate the injected fault current. Throughout the network $\mu \mathrm{P}$ MUs (mirco-phasor measurement units) are employed to estimate linear least squares. Additionally, to minimize the computational hurdle, protective standard devices are used. The feasibility of 134 Bus, $13.8 \mathrm{kV}$ distribution network is validated under noisy and fault measurement. In reference [50], sparse representation vector, machine learning, and clustering of a fuzzy set are used, and single and multiple fault locations are located in a distributive network. This method needs smart meters to know the voltage during the fault and before the fault. To recover the current vector of the fault, a new set of underdetermined equations are formed is used by concatenating the rows of the impedance matrix. The methodology based on the analysis of non-zeroes values and $\mathrm{k}$ nearest neighborhood is used for new machine learning to estimate the fault location. The feasibility of the methodology is confirmed through an implementation of the distribution network under noise-free measurement and noisy condition. In reference [51], the comprehensive sensing technique and smart meters are used to detect fault locations in the distribution network. Current vector is generated through the impedance matrix and voltage sag vector. To solve the optimization issue with or without disturbance (noise), $\log$ barrier algorithm and Primal-Dual Interior Point (PDIP) are employed, respectively. The proposed methodology is validated through simulation under noisy condition. In [52], the new method is presented to locate the single and double wide area of faults in the transmission network. The method required the limited number of buses to find the fault location, and impedance matrix and voltage phasors are used solve equations by sparse representation. The fault zone is located by using non zero elements and sparse current vector. Based on the parameters, line equations are formed to pinpoint the fault location of every fault line. To calculate the current, least square method and substitution theorem are used. Simulation-based results are presented using the 39 Bus system with the noisy condition to validate the concept. To caste estimation problem in sparse vector recovery problem, electrical characteristics are exploited in reference [53]. A new algorithm presented in reference [53] and effectiveness is validated through simulation work on unbalanced distribution network under various operating condition. To attain an optimal place in the number of $\mu \mathrm{P}$ MUs (micro-phasor measurement unit) and to give a unique solution for the state estimation, a difficulty of binary integer linear programming is solved. In reference [54], without using the iterative methodology, the linear equation set is solved by using the new algorithm. The voltage phasors are measured by placing micro-phasor measurement unit at a few buses. The multiplication of the injected vector of current and impedance matrix used the measured voltage vector. Because of single load and generator current, a vector of injection current is sparse when it is compared with total injected current from the grid to the distribution network. The concept is validated through IEEE 123 bus test system and 134 bus real systems $(13.8 \mathrm{kV})$ with the various penetration levels of distributed generators (DG).

\section{Discussion and Outcome}

Based on the literature review on meter placement in power system and power distribution system algorithms, the following conclusions are registered here. The major works on state estimation meter placement in power system carried out until 2006. Very few works related to distribution state estimation meter placement were presented in the past until 2006 due to limited measurement. The listed limitations in distribution system meter placement executed through review are (1) Some techniques fail to derive the global optimal measurement configuration for the distribution network, (2) More measurements are taken for estimating the state of the system, (3) Optimal placement with optimal cost is difficult to achieve, (4) Accuracy level increases with complicated networks, (5) Artificial Intelligence techniques' are not utilized properly to achieve good result and (6) Hybrid techniques' not 
tried in the past to get the output in minimal time. The research work on MP in distribution system kick started in the recent year after the Distributed Generation concepts and the introduction of smart meters in Distribution Networks.

This work presents optimal planning of the metering locations for power distribution system for accurate monitoring and control of distribution management systems which rectifies the maximum number of the above limitations. The algorithm for meter placement was written in mathematical and artificial intelligent techniques. The proposed algorithm in PSO and DE are tested with radial and meshed networks with different constraints. The algorithms have been tested on IEEE systems and TNEB distribution feeders. Figure 3 presents the overall analysis of the proposed hybrid estimator in conversion to percentage for error accuracy and complete performance, which includes time, cost, observability, complexity, constraints and loss of measurements. The effectiveness of the hybrid swarm tuned ANN estimator is evident, where the error percentage has decreased and the performance graph has increased with other algorithms.

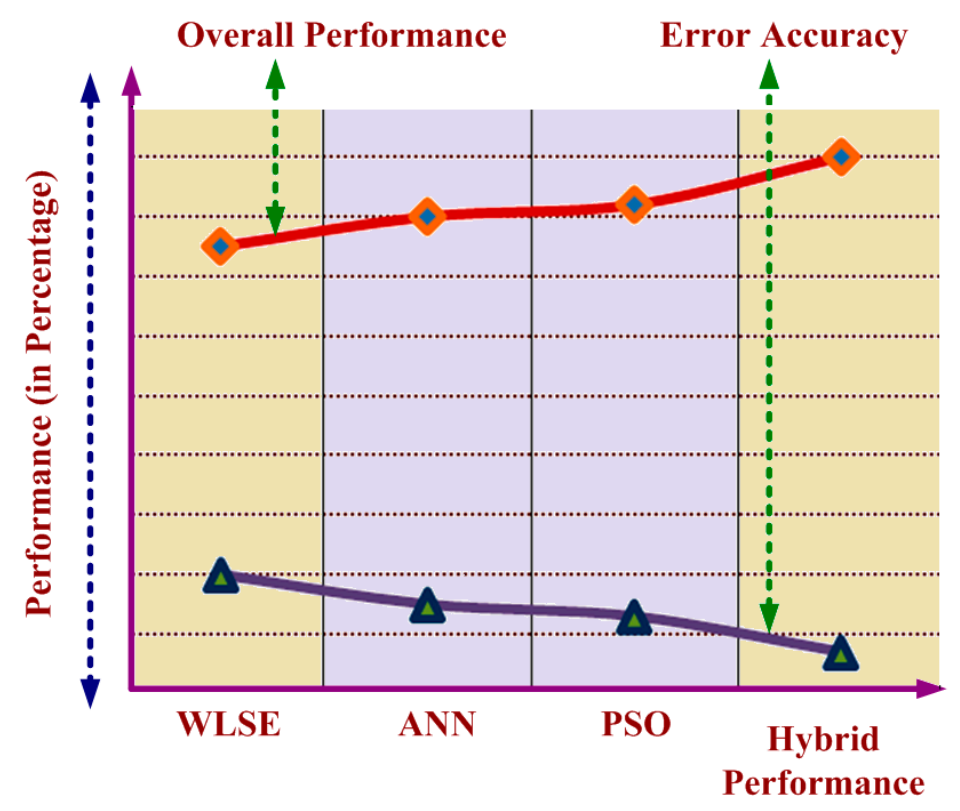

Figure 3. Performance Analysis of Algorithms.

The future work is focused towards the planning optimal number and location of smart meter including phasor measurement units for interconnected microgrids. It is advice to have good feasible algorithm for meter placement in operation of smart cities including all the constraints of energy requirements. The multi objective algorithm needs to be developed, which would satisfy all the meter placement problems like RTU Placement, Harmonic MP, Voltage Sag MP, Power Quality MP, PMU MP and Smart MP.

\section{Conclusions}

It is essential in the present condition to develop the smart intelligent estimator for electric power utility industry with distributed generation and smart meters. The summarized review in this work on modern power system state estimation meter placement will be utilized for current researchers on meter placement to develop efficient algorithms. There is a huge scope in meter placement and state estimation with injection of DG and interconnected microgrids.

Author Contributions: All authors are involved developing the concept to make the article error free technical outcome for the set investigation work.

Funding: No funding received for this research investigation. 
Acknowledgments: We authors like to acknowledge the Center for Bioenergy and Green Engineering, Department of Energy Technology, Aalborg University, Esbjerg, Denmark for supporting this research activity for the insight technical guidance and providing technical information for this project.

Conflicts of Interest: The authors declare no conflict of interest.

\section{References}

1. Billinton, R.; Li, W. Reliability Assessment of Electric Power Systems Using Monte Carlo Methods; Springer: Boston, MA, USA, 1994.

2. Park, Y.M.; Moon, Y.H.; Choo, J.B.; Kwon, T.W. Design of reliable measurement system for state estimation. IEEE Trans. Power Syst. 1988, 3, 830-836. [CrossRef]

3. Celik, M.K.; Liu, W.H.E. A meter placement algorithm for the enhancement of state estimation function in an energy management system. In Proceedings of the 7th IEEE Mediterranean Electrotechnical Conference, Antalya, Turkey, 12-14 April 1994; pp. 873-876.

4. Abbasy, N.H. Neural network aided design for metering system of power system state estimation. In Proceedings of the IEEE Canadian Conference on AFRICON'96, Stellenbosch, South Africa, 26-27 May 1996; Volume 2, pp. 741-744.

5. Abur, A.; Magnago, F.H. Optimal meter placement for maintaining observability during single branch outages. IEEE Trans. Power Syst. 1999, 14, 1273-1278. [CrossRef]

6. Mori, H.; Sone, Y. Tabu search based meter placement for topological observability in power system state estimation. In Proceedings of the 1999 IEEE Transmission and Distribution Conference (Cat. No. 99CH36333), New Orleans, LA, USA, 11-16 April 1999; Volume 1, pp. 172-177.

7. Koutsoukis, N.C.; Manousakis, N.M.; Georgilakis, P.S.; Korres, G.N. Numerical observability method for optimal phasor measurement units placement using recursive Tabu search method. Transm. Distrib. IET Gener. 2013, 7, 347-356. [CrossRef]

8. Magnago, F.H.; Abur, A. A unified approach to robust meter placement against loss of measurements and branch outages. IEEE Trans. Power Syst. 2000, 15, 945-949. [CrossRef]

9. Donmez, B.; Abur, A. A Computationally Efficient Method to Place Critical Measurements. IEEE Trans. Power Syst. 2011, 26, 924-931. [CrossRef]

10. De Souza, J.C.S.; Filho, M.B.D.C.; Schilling, M.T.; de Capdeville, C. Optimal metering systems for monitoring power networks under multiple topological scenarios. IEEE Trans. Power Syst. 2005, 20, 1700-1708. [CrossRef]

11. Kumar, A.; Das, B.; Sharma, J. Genetic algorithm-based meter placement for static estimation of harmonic sources. IEEE Trans. Power Deliv. 2005, 20, 1088-1096. [CrossRef]

12. Chen, J.; Abur, A. Placement of PMUs to Enable Bad Data Detection in State Estimation. IEEE Trans. Power Syst. 2006, 21, 1608-1615. [CrossRef]

13. Abbasy, N.H.; Ismail, H.M. A Unified Approach for the Optimal PMU Location for Power System State Estimation. IEEE Trans. Power Syst. 2009, 24, 806-813. [CrossRef]

14. Sodhi, R.; Srivastava, S.C.; Singh, S.N. Multi-criteria decision-making approach for multistage optimal placement of phasor measurement units. Transm. Distrib. IET Gener. 2011, 5, 181-190. [CrossRef]

15. Shahraeini, M.; Ghazizadeh, M.S.; Javidi, M.H. Co-Optimal Placement of Measurement Devices and Their Related Communication Infrastructure in Wide Area Measurement Systems. IEEE Trans. Smart Grid 2012, 3, 684-691. [CrossRef]

16. Kongjeen, Y.; Inrawong, P.; Buayai, K.; Kerdchuen, T. Key Cutting Algorithm Application to Measurement Placement for Power System State Estimation. Energy Procedia 2013, 34, 142-147. [CrossRef]

17. Li, X.; Scaglione, A. Robust Decentralized State Estimation and Tracking for Power Systems via Network Gossiping. IEEE J. Sel. Areas Commun. 2013, 31, 1184-1194. [CrossRef]

18. Weng, Y.; Negi, R.; Ilić, M.D. Graphical model for state estimation in electric power systems. In Proceedings of the 13th IEEE International Conference on Smart Grid Communications (SmartGridComm), Vancouver, BC, Canada, 21-24 October 2013; pp. 103-108.

19. Mallick, S.; Ghoshal, S.P.; Acharjee, P.; Thakur, S.S. Optimal static state estimation using improved particle swarm optimization and gravitational search algorithm. Int. J. Electr. Power Energy Syst. 2013, 52, 254-265. [CrossRef] 
20. Tungadio, D.H.; Numbi, B.P.; Siti, M.W.; Jimoh, A.A. Particle swarm optimization for power system state estimation. Neurocomputing 2015, 148, 175-180. [CrossRef]

21. Ramachandran, B.; Bellarmine, G.T. Improving observability using optimal placement of phasor measurement units. Int. J. Electr. Power Energy Syst. 2014, 56, 55-63. [CrossRef]

22. Ramesh, L.; Choudhury, S.P.; Chowdhury, S.; Crossley, P.A. Electrical Power System State Estimation Meter Placement-A Comparative Survey Report. Electr. Power Compon. Syst. 2008, 36, 1115-1129. [CrossRef]

23. Baran, M.E.; Zhu, J.; Kelley, A.W. Meter placement for real-time monitoring of distribution feeders. IEEE Trans. Power Syst. 1996, 11, 332-337. [CrossRef]

24. De Souza, J.C.S.; Filho, M.B.D.C.; Meza, E.M.; Schilling, M.T. Planning metering systems for power distribution systems monitoring. In Proceedings of the IEEE Bologna Power Tech Conference, Bologna, Italy, 23-26 June 2003; Volume 2, pp. 1-6.

25. Wang, H.; Schulz, N.N. A revised branch current-based distribution system state estimation algorithm and meter placement impact. IEEE Trans. Power Syst. 2004, 19, 207-213. [CrossRef]

26. Das, B. Rule Based Algorithm for Meter Placement and ANN Based Bus Voltage Estimation in Radial Power Distribution System. Electr. Power Compon. Syst. 2005, 33, 449-462. [CrossRef]

27. Shafiu, A.; Jenkins, N.; Strbac, G. Measurement location for state estimation of distribution networks with generation. Transm. Distrib. IEE Proc. 2005, 152, 240-246. [CrossRef]

28. Muscas, C.; Pilo, F.; Pisano, G.; Sulis, S. Optimal Placement of Measurement Devices in Electrical Distribution Systems. In Proceedings of the IEEE Instrumentation and Measurement Technology Conference, Sorrento, Italy, 24-27 April 2006; pp. 1873-1878.

29. Muscas, C.; Pilo, F.; Pisano, G.; Sulis, S. Optimal Allocation of Multichannel Measurement Devices for Distribution State Estimation. IEEE Trans. Instrum. Meas. 2009, 58, 1929-1937. [CrossRef]

30. Cecchi, V.; Yang, X.; Miu, K.; Nwankpa, C.O. Instrumentation and Measurement of a Power Distribution System Laboratory for Meter Placement and Network Reconfiguration Studies. IEEE Trans. Instrum. Meas. 2007, 56, 1224-1230. [CrossRef]

31. Bignucolo, F.; Caldon, R. Optimizing the voltage measurements location for the management of active distribution networks. In Proceedings of the 42nd International Universities Power Engineering Conference (IEEE-UPEC'07), Brighton, UK, 4-6 September 2007; pp. 960-964.

32. Ramesh, L.; Chowdhury, S.P.; Chowdhury, S.; Natarajan, A.A. Planning optimal intelligent metering for distribution system monitoring and control. In Proceedings of the Annual IEEE India Conference, Kanpur, India, 11-13 December 2008; Volume 1, pp. 218-222.

33. Moradi, A.; Fotuhi-Firuzabad, M. Optimal Switch Placement in Distribution Systems Using Trinary Particle Swarm Optimization Algorithm. IEEE Trans. Power Deliv. 2008, 23, 271-279. [CrossRef]

34. Kennedy, J.; Eberhart, R.C. A discrete binary version of the particle swarm algorithm. In Proceedings of the 1997 IEEE International Conference on Systems, Man, and Cybernetics. Computational Cybernetics and Simulation, Orlando, FL, USA, 12-15 October 1997; Volume 5, pp. 4104-4108.

35. Singh, R.; Pal, B.C.; Vinter, R.B. Measurement Placement in Distribution System State Estimation. IEEE Trans. Power Syst. 2009, 24, 668-675. [CrossRef]

36. Ramesh, L.; Chakraborthy, N.; Chowdhury, S.P.; Chowdhury, S. Intelligent DE algorithm for measurement location and PSO for bus voltage estimation in power distribution system. Int. J. Electr. Power Energy Syst. 2012, 39, 1-8. [CrossRef]

37. Haughton, D.A.; Heydt, G.T. A Linear State Estimation Formulation for Smart Distribution Systems. IEEE Trans. Power Syst. 2013, 28, 1187-1195. [CrossRef]

38. Xiang, Y.; Ribeiro, P.F.; Cobben, J.F.G. Optimization of State-Estimator-Based Operation Framework Including Measurement Placement for Medium Voltage Distribution Grid. IEEE Trans. Smart Grid 2014, 5, 2929-2937. [CrossRef]

39. Damavandi, M.G.; Krishnamurthy, V.; Martí, J.R. Robust Meter Placement for State Estimation in Active Distribution Systems. IEEE Trans. Smart Grid 2015, 6, 1972-1982. [CrossRef]

40. Ramesh, L.; Chowdhury, S.P.; Chowdhury, S.; Gaunt, C.T. A Literature Review on Optimum Meter Placement Algorithms for Distribution State Estimation. In Proceedings of the IASTED Conference-Power and Energy Systems, Palma de Mallorca, Spain, 7-9 September 2009; Acta Press: Calgary, AB, Canada, 2009; p. 681.

41. Liu, J.; Tang, J.; Ponci, F.; Monti, A.; Muscas, C.; Pegoraro, P.A. Trade-Offs in PMU Deployment for State Estimation in Active Distribution Grids. IEEE Trans. Smart Grid 2012, 3, 915-924. [CrossRef] 
42. Pegoraro, P.A.; Tang, J.; Liu, J.; Ponci, F.; Monti, A.; Muscas, C. PMU and smart metering deployment for state estimation in active distribution grids. In Proceedings of the 2012 IEEE International Energy Conference and Exhibition (ENERGYCON'12), Florence, Italy, 9-12 September 2012; pp. 873-878.

43. Liu, J.; Ponci, F.; Monti, A.; Muscas, C.; Pegoraro, P.A.; Sulis, S. Optimal placement for robust distributed measurement systems in active distribution grids. In Proceedings of the 2013 IEEE International Instrumentation and Measurement Technology Conference (I2MTC), Minneapolis, MN, USA, 6-9 May 2013; pp. 206-211.

44. Liu, J.; Ponci, F.; Monti, A.; Muscas, C.; Pegoraro, P.A.; Sulis, S. Optimal Meter Placement for Robust Measurement Systems in Active Distribution Grids. IEEE Trans. Instrum. Meas. 2014, 63, 1096-1105. [CrossRef]

45. Echternacht, D.; Linnemann, C.; Moser, A. Optimized positioning of measurements in distribution grids. In Proceedings of the 3rd IEEE PES Innovative Smart Grid Technologies Europe (ISGT Europe), Berlin, Germany, 14-17 October 2012; pp. 1-7.

46. Echternacht, D.; Moser, A. Cost optimal meter placement in low and medium voltagte grids considering stochastic dependencies. In Proceedings of the IEEE PES 4th Innovative Smart Grid Technology, PES ISGT Europe, Lyngby, Denmark, 6-9 October 2013; pp. 1-5.

47. Liu, H.; Chen, Y.; Chuah, M.C.; Yang, J. Towards self-healing smart grid via intelligent local controller switching under jamming. In Proceedings of the 2013 IEEE Conference on Communications and Network Security (CNS), National Harbor, MD, USA, 14-16 October 2013; pp. 127-135.

48. Abid, M.R.; Khallaayoun, A.; Harroud, H.; Lghoul, R.; Boulmalf, M.; Benhaddou, D. A Wireless Mesh Architecture for the Advanced Metering Infrastructure in Residential Smart Grids. In Proceedings of the IEEE Green Technologies Conference (GreenTech), Denver, CO, USA, 4-5 April 2013; pp. 338-344.

49. Majidi, M.; Etezadi-Amoli, M. A New Fault Location Technique in Smart Distribution Networks Using Synchronized/Nonsynchronized Measurements. IEEE Trans. Power Deliv. 2018, 33, 1358-1368. [CrossRef]

50. Majidi, M.; Etezadi-Amoli, M.; Fadali, M.S. A Novel Method for Single and Simultaneous Fault Location in Distribution Networks. IEEE Trans. Power Syst. 2015, 30, 3368-3376. [CrossRef]

51. Majidi, M.; Arabali, A.; Etezadi-Amoli, M. Fault Location in Distribution Networks by Compressive Sensing. IEEE Trans. Power Deliv. 2015, 30, 1761-1769. [CrossRef]

52. Majidi, M.; Etezadi-Amoli, M.; Fadali, M.S. A Sparse-Data-Driven Approach for Fault Location in Transmission Networks. IEEE Trans. Smart Grid 2015, 8, 548-556. [CrossRef]

53. Majidi, M.; Etezadi-Amoli, M.; Livani, H.; Fadali, M.S. Distribution systems state estimation using sparsified voltage profile. Electr. Power Syst. Res. 2016, 136, 69-78. [CrossRef]

54. Majidi, M.; Etezadi-Amoli, M.; Livani, H. Distribution system state estimation using compressive sensing. Int. J. Electr. Power Energy Syst. 2017, 88, 175-186. [CrossRef] 